\title{
Trauma and Posttraumatic Stress Disorder (PTSD) in Patients with Schizophrenia or Schizoaffective Disorder
}

\author{
Miriam J. J. Lommen · Kathleen Restifo
}

Received: 1 July 2008/Accepted: 10 September 2009/Published online: 24 September 2009

(C) The Author(s) 2009. This article is published with open access at Springerlink.com

\begin{abstract}
Trauma and posttraumatic stress disorder (PTSD) have high prevalence among individuals with severe mental illness, such as schizophrenia or schizoaffective disorder. This study examined whether trauma and PTSD are under-detected in this population, and whether the cognitive theory of PTSD is applicable to these individuals. Traumatic experiences, PTSD symptoms and negative posttraumatic cognitions were directly measured with questionnaires, and compared to information obtained via chart-review. Results showed clear evidence of underreport of trauma and under-diagnosis of PTSD in patients' charts. Furthermore, negative posttraumatic cognitions were positively related to PTSD symptom severity, supporting the cognitive model of PTSD. These findings underscore the importance of assessing trauma history as well as PTSD in the routine evaluation of patients with schizophrenia or schizoaffective disorder in outpatient clinical settings. Furthermore, the finding of negative posttraumatic cognitions suggests that the cognitive model of PTSD may be applicable to patients with schizophrenia or schizoaffective disorder.
\end{abstract}

Keywords Under-recognition · Trauma .

Posttraumatic stress disorder - Schizophrenia .

Cognitive model of PTSD

M. J. J. Lommen ( ()

Department of Clinical and Health Psychology, Utrecht University, PO Box 80.140, 3508 TC Utrecht, The Netherlands e-mail: M.J.J.Lommen@uu.nl

K. Restifo

Department of Clinical Psychological Science, Maastricht University, PO Box 616, 6200 MD Maastricht, The Netherlands e-mail: K.Restifo@maastrichtuniversity.nl

\section{Introduction}

Trauma and PTSD in Severe Mental Illness

Trauma and posttraumatic stress disorder (PTSD) have high prevalence among individuals with severe mental illness, such as schizophrenia. Between 69.5 and $98 \%$ of this population experienced a traumatic event in their life (Gearon et al. 2003; Mueser et al. 1998; Neria et al. 2002; Resnick et al. 2003), compared to $39-56 \%$ in the general population (Breslau et al. 1991; Kessler et al. 1995). Rates of current PTSD in individuals with a severe mental illness range from 29 to 48\% (Cascardi et al. 1996; Craine et al. 1988; Mueser et al. 1998; Switzer et al. 1999). Rates of current PTSD in studies focusing on schizophrenia specifically found 13, 28 and 29\% (Mueser et al. 1998, 2004; Resnick et al. 2003). These rates are much higher than the prevalence of lifetime PTSD in the general population which ranges from 7.8 to 9.2\% and the point prevalence of 2\% (Breslau et al. 1991; Kessler et al. 1995; Stein et al. 1997).

Although trauma and PTSD are common among individuals with a severe mental illness, clinicians frequently overlook trauma and PTSD. A recent study by Howgego et al. (2005) found that 33\% of their sample of patients of a community mental health setting with a severe mental illness met the diagnostic criteria for PTSD. Only $4 \%$ of the sample had a formal diagnosis of PTSD in their medical record. The general lack of recognition and documentation of trauma and PTSD has been demonstrated in a number of studies (Callcott et al. 2004; Craine et al. 1988; Davidson 2001; Davidson and Smith 1990; McFarlane et al. 2001; Mueser et al. 1998; Rose et al. 1991; Zimmerman and Mattia 1999).

The need for recognition of trauma and PTSD is important among patients with a severe mental illness, as 
previous studies show that trauma among these patients is related to higher rates of substance abuse, higher risk of relapse, and higher costs of psychiatric services, such as hospitalizations (Beck and van der Kolk 1987; Briere et al. 1997; Bryer et al. 1987; Goff et al. 1991; McFarlane et al. 2001; Mueser et al. 2002, 2004; Rose et al. 1991). The experience of a traumatic event can be considered as a psychosocial stressor, which increases the level of schizophrenic symptoms (Mueser et al. 2002). Furthermore, a study by Vogel et al. (2006) of inpatients with schizophrenia, found that posttraumatic symptomatology rather than trauma itself, increased the risk of greater current psychopathological distress. PTSD may be expected to worsen the course of the severe mental illness and may contribute to substance abuse, psychiatric and medical comorbidity, and psychiatric and health service utilization. Finally, as long as PTSD is not recognized, it cannot be treated and may continue its negative effect on both physical as well as mental health outcome (Hidalgo and Davidson 2000; Mueser et al. 2004).

\section{Relationships Between Trauma, PTSD and Psychotic Disorders}

Although trauma and PTSD are more prevalent in people with a psychosis compared to the general population, the precise relationship between trauma, PTSD and psychosis is still unclear. Three different kinds of possible relationships seem most likely. First, psychosis can cause PTSD. According to the traditional view, a traumatic event must include actual or threat, death, serious injury, or threat to physical integrity. However, this threat may be subjectively as well as objectively experienced: for example, a psychosis can have the ability to threaten one's own or others' life. A study by Kilpatrick et al. (1989) supports the validity of subjective interpretations of threat, showing that the way assault was perceived by their victims was predictive of the development of PTSD, regardless of the objective threat like use of weapons. Moreover, people may display the full PTSD symptoms without the experience of a direct experience of acute precipitating trauma similar to those experiencing more catastrophic trauma (Morrison et al. 2003). A growing number of research studies suggests that the experience of a first psychosis and its treatment can be traumatic and leading to PTSD-like problems (McGorry et al. 1991; Mueser and Rosenberg 2003; Shaner and Eth 1989; Williams-Keeler et al. 1994). Further, a review of Morrison et al. (2003) shows that many studies using different methodologies found consistent high rates of PTSD in response to psychosis. These findings make it reasonable to conclude that some people do develop PTSD as a response to psychotic experiences (Morrison et al. 2003).
Secondly, trauma can cause psychosis. As many people with psychotic symptoms may have endured experiences of trauma prior to the onset of their psychosis, especially childhood sexual and physical abuse, traumatic life events may contribute to the development of psychosis (Morrison et al. 2003). A review on childhood trauma and psychotic disorders showed that the prevalence of reported childhood traumas ranged between 28 and $73 \%$, with childhood sexual abuse ranging from 13 to $61 \%$ and childhood physical abuse ranging from 10 to $61 \%$ (Bendall et al. 2007). Although the exact role of trauma is still unknown, childhood abuse seems to be implicated in the development of psychosis for a substantial portion of patients. However, more research is required to draw definite conclusions (Bendall et al. 2007; Friedman and Tin 2007; Morrison et al. 2003; Morgan and Fisher 2007).

Thirdly, psychosis and PTSD can both be part of a spectrum of responses to a traumatic event. There is some evidence for a common developmental process, as factors such as dissociation or attribution style may mediate the development of psychosis as well as PTSD following a traumatic event (Morrison et al. 2003). Moreover, the symptom similarity of these two disorders suggests the possibility that they may be similar entities, which may be part of a spectrum of responses to a traumatic event (Morrison et al. 2003). Nevertheless, more research studies are required for a more detailed understanding of the relationships between trauma, PTSD and psychosis.

\section{Treatment of PTSD in Schizophrenia or Schizoaffective Disorder}

Since both trauma and PTSD are overrepresented among individuals with a psychotic disorder, treatment of PTSD in this population may be an important but often overlooked component. Recently, Mueser et al. (2007) studied the effectiveness of a cognitive-behavioural program for PTSD in persons with a severe mental illness, including schizophrenia and schizoaffective disorder. Results showed that this treatment contributed to clinical improvement in PTSD as well as improvement in other symptoms. The cognitivebehavioural treatment is based on the cognitive model of PTSD, in which PTSD is seen as a normal reaction to a traumatic event (Ehlers and Clark 2000). Ehlers and Clark (2000) hypothesized that PTSD becomes persistent when individuals process the trauma in a way that leads to a sense of current threat. This sense of threat arises as a consequence of excessively negative appraisals of the trauma and/or its sequelae on the one hand and a disturbance of autobiographical memory characterized by poor elaboration and contextualization, strong associative memory and strong perceptual priming on the other hand. Those negative appraisals maintain PTSD by directly 
producing negative emotions, and by encouraging individuals to engage in dysfunctional coping strategies that have the paradoxical effect of enhancing PTSD symptoms. The negative appraisals about traumatic events and how they are responded to can have major effects on individuals' beliefs or underlying cognitive schemas about themselves, other people, or the world in general. Changes in the negative appraisals and the trauma memory are prevented by a series of problematic behavioural and cognitive strategies. According to this theory, a wide range of cognitive-behavioural interventions are useful to modify these factors that have contributed to the maintenance of PTSD. Many studies in non-psychiatric populations support this cognitive model of PTSD (Clohessy and Ehlers 1999; Dunmore et al. 1999; Ehlers et al. 2005, 1998; Halligan et al. 2003; Steil and Ehlers 2000). Although the study of Mueser et al. (2007) showed that changes in trauma-related cognitions may mediate changes in PTSD symptoms in people with a severe mental illness, it is questionable if these results can be generalized to specifically the group with schizophrenia or schizoaffective disorder which was represented by only ten participants (12\%). Therefore, it is still unclear whether the cognitive model of PTSD can be applied to people with schizophrenia or schizoaffective disorder.

\section{Aims of the Present Study}

The aim of this study is to examine whether trauma and PTSD are under-reported in a population of psychotic patients. A second aim is to investigate whether the cognitive theory of PTSD can be partially generalized to people with schizophrenia or schizoaffective disorder. The first hypothesis of this study is that lifetime traumatic events are more prevalent among people with schizophrenia or schizoaffective disorder in an outpatient clinical setting than in the general population, as measured in other studies. As we expect to find that trauma is under-reported in this population, the second hypothesis supposes that lifetime trauma experiences are higher when measured directly with questionnaires than when measured with information obtained with chart-review. Thirdly, we expect to find a higher prevalence of PTSD in people with schizophrenia or schizoaffective disorder than in the general population, as measured in other studies. Fourthly, we expect to find that PTSD is under-diagnosed in this population, as manifested by a higher rate of PTSD using a selfreport questionnaire than using chart-review. Finally, we expect that PTSD symptom severity will be positively related to negative posttraumatic cognitions in this sample, supporting the applicability of the cognitive model of PTSD to people with schizophrenia or schizoaffective disorder. If our hypotheses are confirmed, there will be implications for both routine assessments of trauma and PTSD in individuals with schizophrenia or schizoaffective disorder. Confirmation of our last hypothesis would support the extension of the cognitive model of PTSD to this population, and would suggest that treatment based on this model might be beneficial in this population.

\section{Methods}

\section{Participants}

Thirty-three patients recruited from an outpatient clinical setting in Sittard, the Netherlands, participated in this study. This sample consisted of 23 men and 10 women, who received the primary diagnosis of schizophrenia ( $N=23)$ or schizoaffective disorder $(N=10)$ according to their charts. Participants were in the age of 21-63 $(M=$ 42.3, $\mathrm{SD}=10.6$ ).

\section{Instruments}

Prior research suggests that assessment tools for trauma and PTSD developed for the general population are appropriate for use among people with schizophrenia (Goodman et al. 1999; Mueser et al. 2001, 2004; Resnick et al. 2003). Based on these findings, the following measures were used.

The Trauma History Questionnaire-Revised (THQ-R) was used to assess experiences of traumatic events in childhood as well as over lifetime (Mueser et al. 1998). This self-report questionnaire consists of 16 items, which are rated as 0 (no) or 1 (yes). All items met the DSM-IV criteria $\mathrm{A}_{1}$ about the objective threat of the traumatic experience. This questionnaire was successfully used in previous studies of trauma and severe mental illness (Mueser et al. 1998, 2007; Resnick et al. 2003). Total score ranges from 0 to 16 . The internal consistency in this sample was Cronbach's $\alpha=.65$.

The severity of posttraumatic stress disorder was measured by the PTSD Symptom Scale, self report (PSS-SR; Foa et al. 1993). The PSS-SR asked participants to rate how often they experienced each of the 17 PTSD symptoms specified in DSM-IV (American Psychiatric Association 1994; Table 1) during the last month, ranging from 0 (not at all/only one time) to 3 (5 or more times a week/ almost always). The sum of these scores represented the severity of PTSD symptoms. Total scores ranges from 0 to 51. Foa et al. (1993) reported satisfactory internal consistency, high test-retest reliability, and good concurrent validity with other PTSD measures. The internal consistency in this sample was Cronbach's $\alpha=.88$. The individual was also asked to give a short description of their 
worst traumatic experience in order to determine if the participant met the $A_{1}$ criteria for exposure to a traumatic event specified in DSM-IV (APA 1994; Table 1). To investigate the patient's response to the traumatic event, three self-report questions assessing intense fear, helplessness, and horror were added (criteria $\mathrm{A}_{2}$ ). Further questions were posed about the duration of the symptoms and the impairments these symptoms caused in order to investigate whether the person met the $\mathrm{E}$ and $\mathrm{F}$ criteria for PTSD specified in DSM-IV (APA 1994; Table 1). To assess whether the participant met the criteria for PTSD, two different scoring rules were used. One scoring a symptom as present if it was rated as at least 1 (once a week or less/once in a while), and another more conservative scoring rule scoring a symptom as present if it was rated at least 2 (2-4 times a week/half the time).

To assess participants' thoughts and beliefs the Post Traumatic Cognitions Inventory (PTCI) was used (Foa et al. 1999). This 33-itemed self report questionnaire contained three subscales, namely negative cognitions about self (21 items), negative cognitions about the world
(7 items) and self-blame for the trauma (5 items). Answers were rated on a 7-point scale ranging from 1 ("totally disagree") to 7 ("totally agree"). Total score ranges from 33 to 231. The PTCI was successfully used in a previous study of trauma and severe mental illness (Mueser et al. 2007). In the present sample, the Cronbach's alphas for the whole questionnaire, the subscale negative self-cognitions, negative cognitions about the world and self-blame were respectively, .92, .92, .74 and .68.

Demographic information was collected using information in the patients' charts, including age, gender, primary diagnosis and medication.

\section{Procedure}

The study was conducted between July 23, 2007 and November 6, 2007. The target group consisted of patients who were in care of an outpatient clinical setting in Sittard, the Netherlands, who had schizophrenia or schizoaffective disorder as primary diagnosis. According to their charts, 173 patients satisfied this criterion (Fig. 1). All primary

Table 1 Diagnostic criteria posttraumatic stress disorder (PTSD) from DSM-IV

A. The person has been exposed to a traumatic event in which both of the following were present

(1) The person experienced, witnessed, or was confronted with an event or events that involved actual or threatened death or serious injury, or a threat to the physical integrity of self or others

(2) The person's response involved intense fear, helplessness, or horror

B. The traumatic even is persistently re-experienced in one (or more) of the following ways

(1) Recurrent and intrusive distressing recollections of the event, including images, thoughts, and/or perceptions

(2) Recurrent distressing dreams of the event

(3) Acting or feeling as if the traumatic event were recurring (includes a sense of reliving the experience, illusions, hallucinations, and/or dissociative flashback episodes, including those that occur on awakening or when intoxicated)

(4) Intense psychological distress at exposure to internal or external cues that symbolize or resemble an aspect of the traumatic event

(5) Physiological reactivity on exposure to internal or external cues that symbolize or resemble an aspect of the traumatic event

C. Persistent avoidance of stimuli associated with the trauma and numbing of general responsiveness (not present before the trauma), as indicated by at least three of the following

(1) Efforts to avoid thoughts, feelings, and/or conversations associated with the trauma

(2) Efforts to avoid activities, places, and/or people that arouse recollections of the trauma

(3) Inability to recall an important aspect of the trauma

(4) Markedly diminished interest or participation in significant activities

(5) Feeling of detachment or estrangement from others

(6) Restricted range of affect (e.g., inability to have loving feelings)

(7) Sense of a foreshortened future (e.g., does not expect to have a career, marriage, children, or a normal life span)

D. Persistent symptoms of increased arousal (not present before the trauma), as indicated by at least two of the following

(1) Difficulty falling or staying asleep

(2) Irritability or outbursts of anger

(3) Difficulty concentrating

(4) Hypervigilance

(5) Exaggerated startle response

E. Duration of the disturbance (symptoms in Criteria B, C, and D) is more than one (1) month

F. The disturbance causes clinically significant distress and/or impairment in social, occupational, and/or other important areas of functioning 
therapists of these patients were approached. They were informed about the study and the procedure. After that, they were requested to make a selection of patients who met the inclusion criteria and to ask those patients for permission to report their names to the researcher. The inclusion criteria were age of at least 18 years, Dutch speaking and able to understand the questions. Exclusion criteria were severe medical problems preventing participation, insufficient mastery of the Dutch language, too florid psychotic condition or too chaotic speech preventing sufficient communication, and exclusion of the patients by the primary therapists themselves. Selected patients who gave their permission were contacted by telephone or during their visit at the setting. If they were interested in participation, an appointment was made. The majority of the appointments took place in the outpatient clinical setting, but when necessary, the researcher visited patients at their homes. In all situations, the patient and researcher were alone in one room during the research. After the study was described to the participants, written informed consent was obtained from all patients preceding participation in the study.

The participants completed the three self-report questionnaires in the presence of the researcher and answered the three open questions, which were added to the PSS-SR to assess the DSM-IV criteria for PTSD (APA 1994; Table 1). All questions of the self-report questionnaires were read out loud. After each question, participants rated their answer. In this way, the researcher had the opportunity to detect concentration problems and to offer

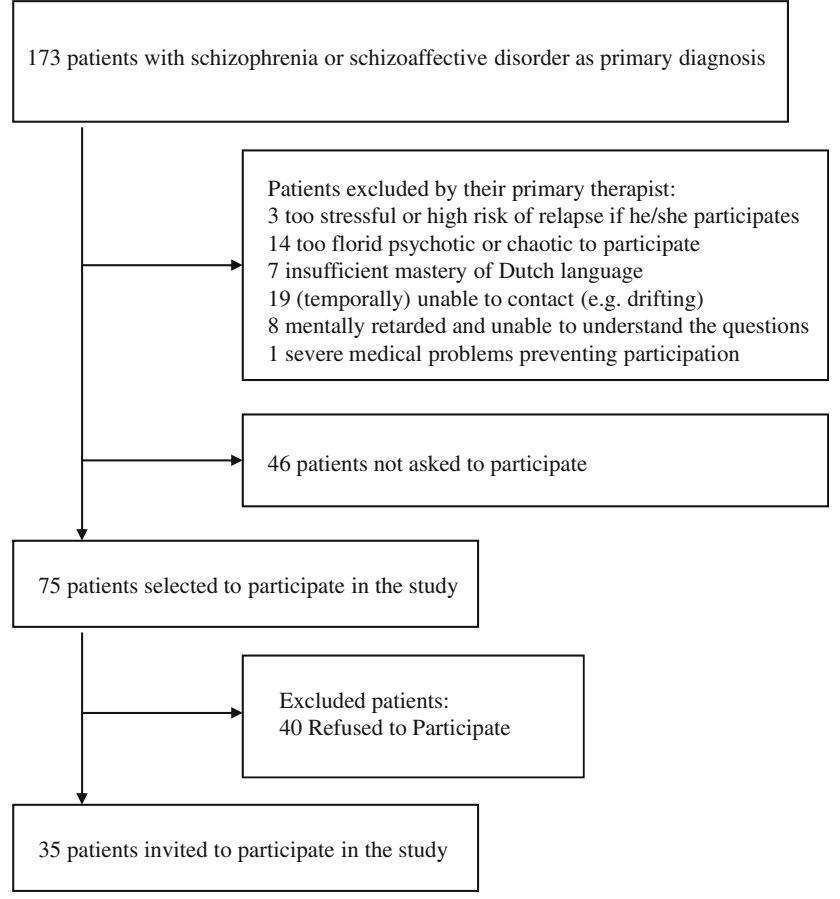

Fig. 1 Recruitment stages participants a short break. In addition, this manner of testing made it easier for the participants to ask for explanation if there was something unclear to them. The duration of the questionnaires varied between thirty and ninety minutes.

Chart review included all available information in the electronic charts of patients. Old paper charts were also included, as these were already scanned into the electronic charts. The present diagnosis of the patient was also available in the chart. To determine if a specific traumatic event was reported in the chart, parts of the chart that normally report information about traumatic events were read, furthermore all information available was searched for words including 'trauma', and other words specifically related to the event itself.

\section{Data-Analysis}

Analyses were done with SPSS version 15.0. First of all, descriptive statistics (proportions, mean, and standard deviation) were calculated. Skewness and Kurtosis were calculated in order to check if the variables were normally distributed. Those deviating from normality were transformed using the square root (scores on the PTCI subscale negative self and whole PTCI). Independent sample $t$-test determined the difference in THQ-R total score between men and women. Chi-Square tests were used to determine the differences in gender on the individual THQ-R items. Linear regression analyses were conducted to determine the relationship between posttraumatic cognitions and PTSD symptom severity. In order to determine which proportion each subscale of the PTCI explained in PTSD symptom severity, backward regression analyses with $P<.05$ criterion including the PTCI subscales were run and Pearson correlations were calculated among the PTCI scales and PTSD symptom severity. Additional linear regression analyses were run to identify confounding and interactive effects of gender and diagnosis.

The authors certify responsibility for this study and have no known conflicts of interest.

\section{Results}

Prevalence of Lifetime Traumatic Experiences

To test the hypothesis that lifetime traumatic events are more common among people with schizophrenia or schizoaffective disorder than in the general population, the Trauma History Questionnaire-Revised (THQ-R) was used. Results are presented in Table $2.97 .0 \%$ of the participants reported having experienced at least one traumatic event during their lifetime, $81.8 \%$ at least two, and $60.6 \%$ at least 
three. The most common traumatic experience in this sample was the sudden and unexpected death of a close friend or loved one due to an accident, illness, suicide or murder $(N=23,69.7 \%)$, followed by the threat of death or serious body harm $(N=13,39.4 \%)$ and stalking causing fear or worries about own safety $(N=12,36.4 \%)$. Independent sample $t$-test showed no significant difference in the number of lifetime traumatic events between women and men $(t=-.06, d f=31, P=.759)$, although ChiSquare tests showed that women reported significantly more unwanted sexual contact after 16th birthday than men $\left(\chi^{2}=4,320, d f=1, P=.038\right)$.

\section{Trauma According to Chart-Review}

In order to test whether the rate of lifetime trauma experiences were higher when measured with questionnaires than measured with information obtained with chart-review as stated by the second hypothesis, the description of the worst event that ever happened, assessed with the additional question to the PTSD Symptom Scale-self report (PSS-SR), was used (see Methods). Of these worst events, $69.7 \%$ were documented in the participants' charts.

\section{Prevalence of Posttraumatic Stress Disorder}

To test the third hypothesis that the PTSD prevalence in people with schizophrenia or schizoaffective disorder is higher than in the general population, the PTSD Symptom
Scale-self report was used. There are several ways to determine the rate of current PTSD in this sample and two different scoring rules were used. Because the DSM-IV criteria $A_{1}$ of an objective threat may be less relevant in this population (as will be further discussed), rates including and excluding this criteria were determined. Including only the $\mathrm{A}_{2}$ to $\mathrm{F}$ criteria according to the DSM-IV (APA 1994; Table 1), using the scoring rule that a symptom is present if it is rated at least as 1 (once a week or less/once in a while), $39.4 \%$ of the participants met the criteria for current PTSD. When DSM-IV criteria $A_{1}$ was also included, so only traumas with an objective threat were included, the prevalence decreased to $18.2 \%$. Using the conservative scoring rule scoring a symptom as present if it was rated at least 2 (2-4 times a week/half the time) and excluding criteria $\mathrm{A}_{1}$, the number of participants meeting the criteria for current PTSD at the time of the study was $21.2 \%$. The inclusion of criteria $A_{1}$ led to a decrease to $9.1 \%$. A chart-review tested the fourth hypothesis supposing PTSD rates to be higher using a self-report questionnaire than using a chart-review. Results showed that none of the participants had PTSD diagnosis in their charts.

\section{Posttraumatic Cognitions Related to PTSD Symptom Severity}

To test the hypothesis that PTSD symptom severity is positively related to negative posttraumatic cognitions in people with schizophrenia or schizoaffective disorder,
Table 2 Traumatic experiences in the sample assessed by the THQ-R

\begin{tabular}{|c|c|c|c|}
\hline & $\begin{array}{l}\text { Males } \\
(N=23) \\
N(\%)\end{array}$ & $\begin{array}{l}\text { Females } \\
(N=10) \\
N(\%)\end{array}$ & $\begin{array}{l}\text { Total } \\
(N=33) \\
N(\%)\end{array}$ \\
\hline Motor vehicle accident & $6(26.1)$ & $3(30.0)$ & $9(27.3)$ \\
\hline Other accidents & $3(13.0)$ & $1(10.0)$ & $4(12.1)$ \\
\hline Warfare or combat & $1(4.3)$ & 0 & $1(3.0)$ \\
\hline $\begin{array}{l}\text { Sudden and unexpected death of a close friend } \\
\text { or loved one }\end{array}$ & $16(69.6)$ & $7(70.0)$ & $23(69.7)$ \\
\hline Robbery involving a weapon & $4(17.4)$ & 0 & $4(12.1)$ \\
\hline Been hit or beaten up and badly hurt by a stranger & $6(26.1)$ & $3(30.0)$ & $9(27.3)$ \\
\hline Witnessed someone seriously injured or killed & $5(21.7)$ & $2(20.0)$ & $7(21.2)$ \\
\hline Threat of death or serious body harm & $9(39.1)$ & $4(40.0)$ & $13(39.4)$ \\
\hline Childhood physical abuse & $2(8.7)$ & 0 & $2(6.1)$ \\
\hline Witness to family violence & $4(17.4)$ & 0 & $4(12.1)$ \\
\hline Intimate partner abuse & $3(13.0)$ & $3(30.0)$ & $6(18.2)$ \\
\hline $\begin{array}{l}\text { Before 16th birthday, unwanted sexual contact } \\
\text { with someone at least } 5 \text { years older }\end{array}$ & $3(13.0)$ & $1(10.0)$ & $4(12.1)$ \\
\hline $\begin{array}{l}\text { Before 16th birthday, unwanted sexual contact } \\
\text { with someone about the same age }\end{array}$ & $4(17.4)$ & $2(20.0)$ & $6(18.2)$ \\
\hline After 16th birthday, unwanted sexual contact & $1(4.3)$ & $3(30.0)$ & $4(12.1)$ \\
\hline Stalking & $7(30.4)$ & $5(50.0)$ & $12(36.4)$ \\
\hline $\begin{array}{l}\text { Any other events that were life threatening, caused } \\
\text { serious injury, or were highly disturbing or distressing }\end{array}$ & $12(52.2)$ & $4(40.0)$ & $16(48.5)$ \\
\hline
\end{tabular}


linear regression analysis was used. The total score on the Post Traumatic Cognitions Inventory (PTCI) was significantly related to PTSD symptom severity as assessed with the PSS-SR $(r=.74, P<.001)$. Additional linear regression analyses were conducted with the scores on the PTCI subscales put in separately to investigate if all three kinds of negative cognitions were related to PTSD symptom severity. Negative cognitions about self, negative cognitions about the world as well as self-blame were significantly positive related to PTSD symptom severity. Results are presented in Table 3.

To control for content overlap of the predictor variables, backward regression analysis including the PTCI subscales were run and the Pearson correlations among the subscales were calculated. The backward regression analysis led to the exclusion of the subscale negative cognitions about the world $(\beta=.14, \quad P=.415)$. Although this subscale $(r=.57, P<.01)$ correlated higher with PTSD symptom severity than the subscale self-blame $(r=.46, P<.01)$, exclusion may be the consequence of the content overlap with the subscale negative cognitions about self $(r=.63$, $P<.01)$ (Table 3).

Compared to the individual subscales of the PTCI, the total score on the PTCI correlated stronger to PTSD symptom severity. Moreover, the explained variation of the model including PTCI total score was higher than that of the model including the PTCI subscales, which remained after backward regression (55.0\%; 52.9\%). Hence, the PTCI total score was used in further analyses.

To identify possible confounding effects of gender $(1=$ male; $2=$ female $)$ and primary diagnosis $(1=$ schizophrenia; $2=$ schizoaffective disorder), these variables were added to the linear regression analyses. The inclusion of one of these variables did not lead to obvious differences in the value $\beta$, so no confounding effects of these variables were found (Table 4).

Linear regression analyses including the interaction variables gender $(\beta=.43, P=.648)$ and primary diagnosis $(\beta=.46, P=.608)$ with PTCI total score, showed no significant interactions of these variables, considering the relationship between posttraumatic cognitions and PTSD symptom severity.
Table 4 Linear regressions with PTSD symptom severity as dependent variable to identify possible confounding effects

\begin{tabular}{lll}
\hline & $\beta_{1}$ & $\beta_{2}$ \\
\hline Model 1 & $.74(P<.001)$ & \\
Model 2 & $.74(P<.001)$ & $.04(P=.773)$ \\
Model 3 & $.75(P<.001)$ & $.06(P=.631)$ \\
\hline
\end{tabular}

Model 1: PTSD symptom severity $=\beta_{0}+\beta_{1} \times$ PTCI total score $+\varepsilon$

Model 2: PTSD symptom severity $=\beta_{0}+\beta_{1} \times$ PTCI total score $+\beta_{2} \times$ gender $+\varepsilon$

Model 3: PTSD symptom severity $=\beta_{0}+\beta_{1} \times$ PTCI total scor$\mathrm{e}+\beta_{2} \times$ primary diagnosis $+\varepsilon$

\section{Discussion}

This study examined the prevalence of trauma and PTSD in patients with schizophrenia or schizoaffective disorder, in an outpatient clinical setting as assessed by questionnaires and compared these with rates found using chart-review and rates found in the general population. Furthermore, we examined the applicability of the cognitive model of PTSD in this population by investigating the association between posttraumatic cognitions and PTSD symptom severity.

The results showed that $97.0 \%$ of the patients reported having experienced at least one traumatic event in lifetime, confirming our hypothesis that the prevalence of lifetime traumatic events in this sample of people with schizophrenia or schizoaffective disorder is higher than in the general population (39-56\%; Breslau et al. 1991; Kessler et al. 1995). The presence of gender differences in the general trauma literature were not found in this study, as the number of experienced traumatic events was not significantly higher for women. However, on one specific trauma significant gender differences were found: women reported experiencing more unwanted sexual contact after age 16 than men. These findings correspond partially with previous studies reporting gender differences in traumatic experiences among severe mental illness samples. However, this study did not show that women significantly experienced more sexual abuse as a child and men were more likely attacked with a weapon and witnessed a killing
Table 3 Pearson correlations among PTSD symptom severity and the posttraumatic cognitions

* Correlation is significant at the .05 level (2-tailed)

** Correlation is significant at the .01 level (2-tailed)

\begin{tabular}{|c|c|c|c|c|c|}
\hline & 1 & 2 & 3 & 4 & 5 \\
\hline 1. PTSD symptom severity & 1 & & & & \\
\hline 2. Total score on PTCI & $.74 * *$ & 1 & & & \\
\hline $\begin{array}{l}\text { 3. Score on PTCI subscale } \\
\text { negative cognitions about self }\end{array}$ & $.67 * *$ & $.94 * *$ & 1 & & \\
\hline $\begin{array}{l}\text { 4. Score on PTCI subscale negative } \\
\text { cognitions about the world }\end{array}$ & $.57 * *$ & $.81 * *$ & $.63 * *$ & 1 & \\
\hline 5. Score on PTCI subscale self-blame & $.46^{* *}$ & $.52 * *$ & .28 & $.41 *$ & 1 \\
\hline
\end{tabular}


or serious injury of another more frequently, as found in previous studies (Kessler et al. 1995; Mueser et al. 1998, 2007). Our findings may differ from results of prior research due to the small sample size.

The results also confirm our second hypothesis, that lifetime trauma experiences are higher when measured with a questionnaire than measured with information obtained with chart-review. Chart-review determined that only $69.7 \%$ of the events that were experienced by the patients as worst event in their life were reported in their charts. In conclusion, these findings indicate that lifetime traumatic events are under-detected in this sample. Note that the chart-review included charts, which were put into the archives. The primary therapists can retrieve these, but it is unclear whether the primary therapists are well acquainted about all information in the archived charts. Hence it is possible that more than $30.3 \%$ of traumas are not known by the therapist.

The results also support the third hypothesis that PTSD is more prevalent among people with schizophrenia or schizoaffective disorder than in the general population, as measured in previous studies. In this sample, 9.1 to $39.4 \%$ of the patients had current PTSD, depending on the scoring rule used and the inclusion or exclusion of DSM-IV criteria $\mathrm{A}_{1}$, while the rates of lifetime PTSD in the general population range from 7.8 to $9.2 \%$ (Breslau et al. 1991; Kessler et al. 1995) and the point prevalence which was determined at $2 \%$ (Stein et al. 1997). By definition, DSM-IV excludes psychotic experiences as meeting the $A_{1}$ criteria for a traumatic event (Table 1). However, many studies suggest that a substantial proportion of people with psychosis develop PTSD in response to their psychotic experiences and/or their hospital treatment (Morrison et al. 2003; Mueser and Rosenberg 2003; Shaner and Eth 1989; Williams-Keeler et al. 1994). In a sample of participants who had been hospitalized following a psychotic episode, $52.3 \%$ met the criteria for post-psychotic PTSD (Shaw et al. 2002). The development of PTSD was associated with the psychological distress of the experience, supporting the view that subjective instead of solely objective threat determines the impact of the traumatic event. The way an event is perceived seems to play a major role in the development of PTSD (Kilpatrick et al. 1989). Therefore, the $\mathrm{A}_{2}$ criteria (participant's response to the event involving intense fear, helplessness, or horror) may be of importance in the determination of PTSD diagnosis, regardless of the (objective) nature of the precipitating traumatic event $\left(\mathrm{A}_{1}\right.$ criteria). According to this argument, the prevalence of current PTSD in the studied sample ranges from 21.2 to $39.4 \%$, depending on the scoring rule. This prevalence is consistent with prior research on current PTSD in patients with schizophrenia or schizoaffective disorder (Mueser et al. 1998; 2004; Resnick et al. 2003).
Although Mueser et al. (1998) found a higher PTSD rate in people with schizoaffective disorder than in schizophrenia, these differences in primary diagnosis were not found in this study. Neither were any gender differences found. Although gender difference is one of the most robust findings in the general PTSD literature, results of earlier studies among men and women with a severe mental illness found no differences (Brewin et al. 2000; Cascardi et al. 1996; Davidson and Smith 1990; McFarlane et al. 2001; Mueser et al. 1998, 2001; Ollf et al. 2007; Switzer et al. 1999). The lack of gender differences in PTSD among patients with schizophrenia or schizoaffective disorder may be due to the overall high rate of trauma exposure in this population.

A reason to believe that the actual prevalence of current PTSD is closer to the $21.2 \%$ than the $39.4 \%$ is the symptom overlap between schizophrenia and PTSD. Participants could have scored higher on certain items of the PSS-SR due to symptoms of their primary diagnosis. For example difficulty with concentrating can be symptom of schizophrenia as well as PTSD. Nevertheless, the diagnosis of PTSD depends on several criteria, so the influence of the symptom overlap on PTSD diagnosis will be limited. Despite the limitation in our ability to draw definite conclusions about the precise prevalence of PTSD in people with schizophrenia or schizoaffective disorder due to the above difficulties differentiating psychotic and post-traumatic symptoms in this population, even the most conservative estimate of the prevalence of current PTSD supported the hypothesis that PTSD prevalence in this group is higher than in the general population.

A chart-review confirmed the fourth hypothesis that rates of PTSD are higher when using a self-report questionnaire than when using a chart-review, because none of the participants in this study received this diagnosis in their charts. In accordance with previous studies, these findings suggest that PTSD is under-diagnosed in this population (McFarlane et al. 2001; Mueser et al. 1998; Zimmerman and Mattia 1999).

Several explanations are suggested to explain this phenomenon of under-detection and under-diagnosis. One of these explanations assumes that PTSD symptoms are often not the presenting complaint, as patients generally will not volunteer this information either because of reluctance to revisit the trauma, or because they are afraid of the clinician's response, or because they simply do not recognize the relevance of any prior trauma to their current problem (Howgego et al. 2005; Jacobsen and Richardson 1987). Moreover, physicians and psychiatrists working with schizophrenic patients may be so focused on assessing psychotic symptoms and the patient's functional level that they do not think about the issue of trauma. There may also be an underlying assumption that psychosis is the main 
problem to focus on in this population. Further, the results may represent poor documentation of assessments and findings. However, they may also be a consequence of change in PTSD symptoms after intake or initial assessment, or patients being more forthcoming, or more comfortable sharing in the study context as opposed to during the clinical consultations with their therapist. Another explanation is the high degree of symptom overlap between PTSD and other diagnoses, contributing to diagnostic confusion when trauma histories are not specifically obtained (Brady et al. 2000). Symptom overlap is especially evident in schizophrenia, as symptoms of schizophrenia may be confused with or contribute to symptoms of PTSD. For example, hallucinations may be confused with flashbacks or other re-experiencing symptoms, and negative symptoms may be confused with avoidant symptoms of PTSD. However, there is evidence that PTSD can be diagnosed reliably among patients who have a severe mental illness (Rosenberg et al. 2001).

The results of this study also support the last hypothesis about the association between posttraumatic cognitions and PTSD symptom severity. Negative posttraumatic cognitions were positively related to PTSD symptom severity. No confounding effects were found of gender or diagnosis, nor significant interaction of these variables. This suggests that there are no significant differences in the relationship between posttraumatic cognitions and PTSD symptom severity according to gender or primary diagnosis in this sample.

Each of the three subscales of the PTCI containing negative cognitions about self, negative cognitions about the world and self-blame for the trauma, correlated positively with PTSD symptom severity. These results support the cognitive model of PTSD of Ehlers and Clark (2000), which suggests that negative appraisals about traumatic events and how they are responded to can have major effects on individuals' beliefs or underlying cognitive schemas about themselves, other people, or the world in general and play a role in the maintenance of PTSD. This relationship has been demonstrated in previous studies, but to the best of the author's knowledge, this was the first study identifying this relationship in a sample of exclusively patients with schizophrenia or schizoaffective disorder, for whom cognitive deficits are part of the disorder. A recent study among people with a severe mental illness showed that changes in trauma-related cognitions may mediate changes in PTSD symptoms in this group as well (Mueser et al. 2007). Together with our findings, these results suggest that the cognitive model of PTSD may be applied to this group, despite the known cognitive deficits among these individuals. These cognitive deficits do not appear to significantly interfere with cognitive therapy, as suggested by the several studies showing positive results about the effectiveness of cognitive-behavioural therapy for the treatment of schizophrenia (Turkington et al. 2008). As the effectiveness of cognitive-behavioural therapy (CBT) in treating PTSD has been shown in many studies among non-psychotic samples and recently in a sample including schizophrenia and schizoaffective disorder (Bisson et al. 2007; Bradley et al. 2005; Mueser et al. 2007; Schnurr et al. 2007; Van Etten and Taylor 1998), this would be an important adjunct treatment for individuals with schizophrenia or schizoaffective disorder and co-morbid PTSD.

\section{Relation Between Trauma, PTSD and Psychosis}

Looking more closely at the seven cases identified with PTSD using the conservative scoring rule and excluding the $\mathrm{A}_{1}$ criteria, three PTSD-related traumas were absolutely non-psychotic and involved objective threat as mentioned in the $\mathrm{A}_{1}$ criteria of the DSM-IV definition of PTSD. In two of the seven cases the PTSD-related trauma happened before the onset of their illness, two after the onset of their illness and in the other cases the trauma was experienced in the same period as the onset of the illness and it remained unclear what happened first. These findings seem to correspond to the three relationships found in previous studies: psychosis can cause PTSD, trauma can cause psychosis, and psychosis and PTSD can both be part of a spectrum of responses to a traumatic event (Morrison et al. 2003).

\section{Limitations}

There are several limitations of this study. First of all, the recruitment procedure could have caused sampling bias, as the approach of patients meeting the inclusion criteria relied upon referrals from the primary therapists of these patients. Therapists may have been reluctant to recommend participation to individuals with severe symptoms or known trauma histories, due to concerns about potential stress of the study. As a consequence, the prevalence of current PTSD may be higher than found in this study.

Another limitation was the use of the primary diagnosis as documented in the charts of the participants, instead of using a structured clinical interview to determine the psychiatric diagnosis. Furthermore, using the self-report questionnaires was a less rigorous way of assessing trauma history, current PTSD and posttraumatic cognitions than using a structured clinical interview. However, in this study the self-report questionnaires were also read aloud to the participants, thus decreasing any possible comprehension problems associated with attention or reading problems; in addition, many other studies have successfully used selfreport questionnaires in comparable samples (Goodman et al. 1999; Mueser et al. 2001, 2007; Resnick et al. 2003). 
Not all internal consistencies of the questionnaires used were satisfying. Firstly, the internal consistency of the THQ-R was with a Cronbach's alpha of .65 weak. The exclusion of the item asking about the experience of a sudden and unexpected death of a close friend or loved one would lead to a higher reliability. However, as such an event can be very traumatic and was experienced by many people is our sample, we decided to maintain this item in the scale. Secondly, the PTCI subscale self-blame had an internal consistency of .68 , which can probably be attributed to the small number of items this scale consisted of. Despite of these two weak reliabilities, all measures that were included in the analyses in order to determine the relationship between the posttraumatic cognitions and PTSD symptom severity, had satisfying to good internal consistencies.

Although participants were asked to report one single traumatic event prior to the questionnaire-part of the PSS, many found it hard to choose one event and reported multiple events. It would have been preferable to assess the PTSD symptoms for every qualifying traumatic event separately. Moreover, chart-review included only the event reported as worst event by the patient. It is unclear whether the findings about under-report can be generalized to other events.

Another limitation is the relatively small sample size of the study, which make the results less reliable. Caution is required when generalizing the conclusions of this study to other groups. It should be noted that nearly all participants were taking a variety of medications, including antipsychotics, anxiolytics, antidepressives and sleep medication. However, medication use will probably not influence the results in a systematic way. Furthermore, it is not feasible to conduct a study of schizophrenia without having the patients who are on multiple medications. Because medications as well as co-morbid disorders were not excluded in this study, the results are more generalizable to other patients with schizophrenia or schizoaffective disorder in outpatient community settings. Finally, because this study was a cross-sectional study, no conclusions can be made about causal relationships between trauma and psychosis. A longitudinal study needs to be done to investigate this.

\section{Clinical Implications}

This study provides evidence of under-report of trauma and under-diagnosis of PTSD in charts of patients with schizophrenia or schizoaffective disorder in a outpatient clinical setting. At the same time, both trauma and PTSD are more prevalent in this group than in the general population. These findings underscore the importance of assessing trauma history as well as PTSD in the routine evaluation of patients with schizophrenia or schizoaffective disorder in outpatient clinical settings, preferably with help of valid assessment techniques. It is important that therapists recognize the prevalence and impact of traumatic experiences in the lives of their patients, considering their negative consequences on mental health outcomes. Improvements in training of physicians, psychiatrists and therapists working with patients with schizophrenia on recognition and detection of trauma and PTSD is recommended to help remedy this problem.

The results concerning the posttraumatic cognitions in relation to PTSD symptom severity support the cognitive model of PTSD, suggesting that treatment based on this model may be effective in treating PTSD in patients with schizophrenia or schizoaffective disorder. Cognitivebehavioural interventions may be able to modify the factors that are contributing to the maintenance of PTSD. Accurate detection and treatment of PTSD may be critical in reducing distress and improving the psychiatric and health functioning of these patients.

\section{Future Research Directions}

More research is needed to develop definite treatment guidelines for PTSD in people with schizophrenia or schizoaffective disorder. Longitudinal studies are needed to prove that posttraumatic negative cognitions play an important role in the maintenance of PTSD in this specific sample. Randomized controlled trials of cognitive-behavioural therapy in this specific group are recommended to determine the effectiveness of the treatment and the improvement on both PTSD as other psychiatric symptoms. Moreover, future studies should focus on the differences between psychotic-related and non-psychoticrelated PTSD and the relation between trauma, PTSD and psychosis. Finally, longitudinal studies are needed to draw more definite conclusions about the causal relationships between trauma and psychosis.

Acknowledgments We thank all participants and therapists of the 'Prins Claus Centrum' in Sittard, who cooperated in this study and supported us with the acquisition of the data. We are grateful for the help of Nancy Keune, Jos Verlinden and Jaap de Bruin, who supported us with the study concept and design. For statistical support, we thank Erik Schouten and Suzanne Lommen. We thank David Bernstein for his advices during the writing of the article. Finally, we want to thank the reviewers for their helpful comments.

Open Access This article is distributed under the terms of the Creative Commons Attribution Noncommercial License which permits any noncommercial use, distribution, and reproduction in any medium, provided the original author(s) and source are credited.

\section{References}

American Psychiatric Association. (1994). Diagnostic and statistical manual of mental disorders (4th ed.). Washington: American Psychiatric Association. 
Beck, J. C., \& van der Kolk, B. A. (1987). Reports of childhood incest and current behavior of chronically hospitalized psychotic women. The American Journal of Psychiatry, 144, 1474-1476.

Bendall, S., Jackson, H. J., Hulbert, C. A., \& McGorry, P. D. (2008). Childhood trauma and psychotic disorders: A systematic, critical review of the evidence. Schizophrenia Bulletin, 34, 568-579.

Bisson, J. I., Ehlers, A., Matthews, R., Pilling, S., Richards, D., \& Turner, S. (2007). Psychological treatments for chronic posttraumatic stress disorder: Systematic review and meta-analysis. The British Journal of Psychiatry, 190, 97-104. doi:10.1192/ bjp.bp.106.021402.

Bradley, R., Greene, J., Russ, E., Dutra, L., \& Westen, D. (2005). A multidimensional meta-analysis of psychotherapy for PTSD. The American Journal of Psychiatry, 162, 214-227. doi:10.1176/ appi.ajp.162.2.214.

Brady, K. T., Killeen, T. K., Brewerton, T., \& Lucerini, S. (2000). Comorbidity of psychiatric disorders and posttraumatic stress disorder. The Journal of Clinical Psychiatry, 61(Suppl 7), 22-32.

Breslau, N., Davis, G. C., Andreski, P., \& Peterson, E. (1991). Traumatic events and posttraumatic stress disorder in an urban population of young adults. Archives of General Psychiatry, 48, 216-222.

Brewin, C. R., Andrews, B., \& Valentine, J. D. (2000). Meta-analysis of risk factors for posttraumatic stress disorder in traumaexposed adults. Journal of Consulting and Clinical Psychology, 68(5), 748-766. doi:10.1037/0022-006X.68.5.748.

Briere, J., Woo, R., McRae, B., Foltz, J., \& Sitzman, R. (1997). Lifetime victimization history, demographics, and clinical status in female psychiatric emergency room patients. The Journal of Nervous and Mental Disease, 185, 95-101. doi:10.1097/0000 5053-199702000-00005.

Bryer, J. B., Nelson, B. A., Miller, J. B., \& Krol, P. A. (1987). Childhood sexual and physical abuse as factors in adult psychiatric illness. The American Journal of Psychiatry, 144, 1426-1430.

Callcott, P., Standart, S., \& Turkington, D. (2004). Trauma within psychosis: Using a CBT model for PTSD in psychosis. Behavioural and Cognitive Psychotherapy, 32, 239-244. doi: 10.1017/S1352465804001249.

Cascardi, M., Mueser, K. T., DeGiralomo, J., \& Murrin, M. (1996). Physical aggression against psychiatric inpatients by family members and partners. Psychiatric Services (Washington, DC), 47, 531-533.

Clohessy, S., \& Ehlers, A. (1999). PTSD symptoms, response to intrusive memories and coping in ambulance service workers. The British Journal of Clinical Psychology, 38, 251-265. doi: 10.1348/014466599162836.

Craine, L. S., Henson, C. E., Colliver, J. A., \& MacLean, D. G. (1988). Prevalence of a history of sexual abuse among female psychiatric patients in a state hospital system. Hospital \& Community Psychiatry, 39, 300-304.

Davidson, J. R. T. (2001). Recognition and treatment of posttraumatic stress disorder. Journal of the American Medical Association, 286(5), 584-590. doi:10.1001/jama.286.5.584.

Davidson, J., \& Smith, R. (1990). Traumatic experiences in psychiatric outpatients. Journal of Traumatic Stress, 3, 459-475. doi: $10.1002 /$ jts. 2490030314.

Dunmore, E., Clark, D. M., \& Ehlers, A. (1999). Cognitive factors involved in the onset and maintenance of posttraumatic stress disorder (PTSD) after physical or sexual assault. Behaviour Research and Therapy, 37, 809-829. doi:10.1016/S0005-7967 (98)00181-8.

Ehlers, A., \& Clark, D. M. (2000). A cognitive model of posttraumatic stress disorder. Behaviour Research and Therapy, 38, 319-345. doi:10.1016/S0005-7967(99)00123-0.
Ehlers, A., Clark, D. M., Hackmann, A., McManus, F., \& Fennell, M. (2005). Cognitive therapy for post-traumatic stress disorder: Development and evaluation. Behaviour Research and Therapy, 43, 413-431. doi:10.1016/j.brat.2004.03.006.

Ehlers, A., Mayou, R. A., \& Bryant, B. (1998). Psychological predictors of chronic posttraumatic stress disorder after motor vehicle accidents. Journal of Abnormal Psychology, 107(3), 508-519. doi:10.1037/0021-843X.107.3.508.

Foa, E. B., Ehlers, A., Clark, D. M., Tolin, D. F., \& Orsillo, S. M. (1999). The posttraumatic cognitions inventory (PTCI): Development and validation. Psychological Assessment, 11(3), 303314. doi:10.1037/1040-3590.11.3.303.

Foa, E. B., Riggs, D. S., Dancu, C. V., \& Rothbaum, B. O. (1993). Reliability and validity of a brief instrument for assessing posttraumatic stress disorder. Journal of Traumatic Stress, 6(4), 459473. doi: $10.1002 /$ jts. 2490060405.

Friedman, T., \& Tin, N. N. (2007). Childhood sexual abuse and the development of schizophrenia. Postgraduate Medical Journal, 83, 507-508. doi:10.1136/pgmj.2006.054577.

Gearon, J. S., Kaltman, S. I., Brown, C., \& Bellack, A. S. (2003). Traumatic life events and PTSD among women with substance use disorders and schizophrenia. Psychiatric Services (Washington, DC), 54(4), 523-528. doi:10.1176/appi.ps.54.4.523.

Goff, D. C., Brotman, A. W., Kindlon, D., Waites, M., \& Amico, E. (1991). Self-reports of childhood abuse in chronically psychotic patients. Psychiatry Research, 37, 73-80. doi:10.1016/0165-1781 (91)90107-Z.

Goodman, L. A., Thompson, K. M., Weinfurt, K., Corl, S., Acker, P., Mueser, K. T., et al. (1999). Reliability of reports of violent victimization and posttraumatic stress disorder among men and women with serious mental illness. Journal of Traumatic Stress, 12(4), 587-599. doi:10.1023/A:1024708916143.

Halligan, S. L., Michael, T., Clark, D. M., \& Ehlers, A. (2003). Posttraumatic stress disorder following assault: The role of cognitive processing, trauma memory, and appraisals. Journal of Consulting and Clinical Psychology, 71, 419-431. doi:10.1037/ 0022-006X.71.3.419.

Hidalgo, R. B., \& Davidson, J. R. (2000). Posttraumatic stress disorder: Epidemiology and health-related considerations. The Journal of Clinical Psychiatry, 61(Suppl 7), 5-13.

Howgego, I. M., Owen, C., Meldrum, L., Yellowlees, P., Dark, F., \& Parslow, R. (2005). Posttraumatic stress disorder: An exploratory study examining rates of trauma and PTSD and its effects on client outcomes in community mental health. BMC Psychiatry, $5(21)$.

Jacobsen, A., \& Richardson, B. (1987). Assault experience of 100 psychiatric inpatients: Evidence of the need for routine inquiry. The American Journal of Psychiatry, 144, 908-913.

Kessler, R. C., Sonnega, A., Bromet, E., Hughes, M., \& Nelson, C. B. (1995). Posttraumatic stress disorder in the National Comorbidity Survey. Archives of General Psychiatry, 52, 1048-1060.

Kilpatrick, D. G., Saunders, B. E., Amick-McMullan, A., Best, C. L., Veronen, L. J., \& Resnick, H. S. (1989). Victim and crime factors associated with the development of crime-related posttraumatic stress disorder. Behavior Therapy, 20, 199-214. doi: 10.1016/S0005-7894(89)80069-3.

McFarlane, A. C., Bookless, C., \& Air, T. (2001). Posttraumatic stress disorder in a general psychiatric inpatient population. Journal of Traumatic Stress, 14(4), 633-645. doi:10.1023/A:10130777 02520.

McGorry, P. D., Chanen, A., McCarthy, E., van Riel, R., McKenzie, D., \& Singh, B. S. (1991). Posttraumatic stress disorder following recent-onset psychosis: An unrecognized postpsychotic syndrome. The Journal of Nervous and Mental Disease, 179, 253258. doi:10.1097/00005053-199105000-00002. 
Morgan, C., \& Fisher, H. (2007). Environment and schizophrenia: Environmental factors in schizophrenia: Childhood trauma-a critical review. Schizophrenia Bulletin, 33(1), 3-10. doi:10.1093/ schbul/sbl053.

Morrison, A. P., Frame, L., \& Larkin, W. (2003). Relationships between trauma and psychosis: A review and integration. The British Journal of Clinical Psychology, 42, 331-351. doi: 10.1348/014466503322528892.

Mueser, K. M., Bolton, E., Carty, P. C., Bradley, M. J., Ahlgren, K. F., DiStaso, D. R., et al. (2007). The trauma recovery group: A cognitive-behavioral program for post-traumatic stress disorder in persons with severe mental illness. Community Mental Health Journal, 43(3), 281-304. doi:10.1007/s10597-006-9075-2.

Mueser, K. T., Goodman, L. B., Trumbetta, S. L., Rosenberg, S. D., Osher, F. C., Vidaver, R., et al. (1998). Trauma and posttraumatic stress disorder in sever mental illness. Journal of Consulting and Clinical Psychology, 66(3), 493-499. doi:10.1037/0022-006X. 66.3.493.

Mueser, K. T., \& Rosenberg, S. D. (2003). Treating the trauma of first episode psychosis: A PTSD perspective. Journal of Mental Health, 12, 103-108.

Mueser, K. T., Rosenberg, S. D., Goodman, L. A., \& Trumbetta, S. L. (2002). Trauma, PTSD, and the course of severe mental illness: An interactive model. Schizophrenia Research, 53, 123-143. doi:10.1016/S0920-9964(01)00173-6.

Mueser, K. T., Salyers, M. P., Rosenberg, S. D., Ford, J. D., Fox, L., \& Carty, P. (2001). Psychometric evaluation of trauma and posttraumatic stress disorder assessments in persons with severe mental illness. Psychological Assessment, 13(1), 110-117. doi: 10.1037/1040-3590.13.1.110.

Mueser, K. T., Salyers, M. P., Rosenberg, S. D., Goodman, L. A., Essock, S. M., Osher, F. C., et al. (2004). Interpersonal trauma and posttraumatic stress disorder in patients with severe mental illness: Demographic, clinical, and health correlates. Schizophrenia Bulletin, 30(1), 45-57.

Neria, Y., Bromet, E. J., Sievers, S., Lavelle, J., \& Fochtmann, L. J. (2002). Trauma exposure and posttraumatic stress disorder in psychosis: Findings from a first-admission cohort. Journal of Consulting and Clinical Psychology, 70(1), 246-251. doi: 10.1037/0022-006X.70.1.246.

Ollf, M., Langeland, W., Draijer, N., \& Gersons, B. P. R. (2007). Gender differences in posttraumatic stress disorder. Psychological Bulletin, 133, 183-204. doi:10.1037/0033-2909.133.2.183.

Resnick, S. G., Bond, G. R., \& Mueser, K. M. (2003). Trauma and posttraumatic stress disorder in people with schizophrenia. Journal of Abnormal Psychology, 112(3), 415-423. doi:10.1037/ 0021-843X.112.3.415.

Rose, S. M., Peabody, C. G., \& Stratigeas, B. (1991). Undetected abuse among intensive case management clients. Hospital \& Community Psychiatry, 42, 499-503.
Rosenberg, S. D., Mueser, K. T., Friedman, M. J., Gorman, P. G., Drake, R. E., Vidaver, R. M., et al. (2001). Developing effective treatments for posttraumatic disorders among people with severe mental illness. Psychiatric Services (Washington, DC), 52(11), 1453-1461. doi:10.1176/appi.ps.52.11.1453.

Schnurr, P. P., Friedman, M. J., Engel, C. C., Foa, E. B., Shea, M. T., Chow, B. K., et al. (2007). Cognitive behavioral therapy for posttraumatic stress disorder in women. JAMA, 297, 820-830.

Shaner, A., \& Eth, S. (1989). Can schizophrenia cause posttraumatic stress disorder? American Journal of Psychotherapy, 4, 588-597.

Shaw, K., McFarlane, A. C., Bookless, C., \& Air, T. (2002). The aetiology of posttraumatic stress disorder following a psychotic episode. Journal of Traumatic Stress, 15(1), 39-47. doi:10.1023/ A: 1014331211311.

Steil, R., \& Ehlers, A. (2000). Dysfunctional meaning of posttraumatic intrusions in chronic PTSD. Behaviour Research and Therapy, 38, 537-558. doi:10.1016/S0005-7967(99)00069-8.

Stein, M. B., Walker, J. R., Hazen, A. L., \& Forde, D. R. (1997). Full and partial posttraumatic stress disorder: Findings from a community survey. The American Journal of Psychiatry, 154, 1114-1119.

Switzer, G. E., Dew, M. A., Thompson, K., Goycoolea, J. M., Derricott, T., \& Mullins, S. D. (1999). Posttraumatic stress disorder and service utilization among urban mental health center clients. Journal of Traumatic Stress, 12, 25-39. doi: 10.1023/A:1024738114428.

Turkington, D., Sensky, T., Scott, J., Barnes, T. R. E., Nur, U., Siddle, R., et al. (2008). A randomized controlled trial of cognitivebehavior therapy for persistent symptoms in schizophrenia: A five-year follow-up. Schizophrenia Research, 98, 1-7. doi: 10.1016/j.schres.2007.09.026.

Van Etten, M. L., \& Taylor, T. (1998). Comparative efficacy of treatments for post-traumatic stress disorder: A meta analysis. Clinical Psychology \& Psychotherapy, 5, 126-144. doi:10.1002/ (SICI) 1099-0879(199809)5:3<126:AID-CPP153>3.0.CO;2-H.

Vogel, M., Spitzer, C., Barnow, S., Freyberger, H. J., \& Grabe, H. J. (2006). The role of trauma and PTSD-related symptoms for dissociation and psychopathological distress in inpatients with schizophrenia. Psychopathology, 39, 236-242. doi:10.1159/000 093924.

Williams-Keeler, L., Milliken, H., \& Jones, B. (1994). Psychosis as precipitating trauma for PTSD: A treatment strategy. The American Journal of Orthopsychiatry, 64, 493-498. doi:10.1037/ h0079543.

Zimmerman, M., \& Mattia, J. I. (1999). Is posttraumatic stress disorder underdiagnosed in routine clinical settings? The Journal of Nervous and Mental Disease, 187, 420-428. doi:10.1097/ 00005053-199907000-00005. 